\title{
The MANGUA Project: A Population-Based HIV Cohort in Guatemala
}

\author{
Juan Ignacio García, ${ }^{1,2}$ Blanca Samayoa, ${ }^{3}$ Meritxell Sabidó, ${ }^{1,4}$ Luis Alberto Prieto, \\ Mikhail Nikiforov, ${ }^{1}$ Rodolfo Pinzón, ${ }^{5}$ Luis Roberto Santa Marina de León, ${ }^{6}$ \\ José Fernando Ortiz, ${ }^{7}$ Ernesto Ponce, ${ }^{8}$ Carlos Rodolfo Mejía, ${ }^{5}$ Eduardo Arathoon, ${ }^{3}$ \\ Jordi Casabona, ${ }^{1,2,9,10}$ and The Mangua Cohort Study Group ${ }^{1}$ \\ ${ }^{1}$ Fundació Sida i Societat, Technical Advisor Unit (UAT), Escuintla National Hospital, 5001 Escuintla, Guatemala \\ ${ }^{2}$ PhD Programme in Methodology of Biomedical Research and Public Health, Department of Paediatrics, Obstetrics, \\ Gynaecology and Preventive Medicine, Universitat Autonoma de Barcelona, Bellaterra, 08193 Catalonia, Spain \\ ${ }^{3}$ Clínica Familiar Luis Ángel García, San Juan de Dios Hospital, Asociación de Salud Integral, 1001 Guatemala City, Guatemala \\ ${ }^{4}$ TransLab, Department of Medical Sciences, University of Girona, Girona, 17004 Catalonia, Spain \\ ${ }^{5}$ Infectious Diseases Clinic, Roosevelt Hospital, 1011 Guatemala City, Guatemala \\ ${ }^{6}$ Juan José Ortega de Coatepeque National Hospital, Coatepeque, 09020 Quetzaltenango, Guatemala \\ ${ }^{7}$ Hospital del Instituto Guatemalteco de Seguridad Social (IGSS), 1009 Guatemala City, Guatemala \\ ${ }^{8}$ National Programme for Prevention and Control of STI and HIV/AIDS (PNS), 1011 Guatemala City, Guatemala \\ ${ }^{9}$ CIBER Epidemiología y Salud Pública (CIBERESP), 28029 Madrid, Spain \\ ${ }^{10}$ Center for Epidemiological Studies on HIV/AIDS and STI of Catalonia (CEEISCAT), Institut Català d'Oncologia, \\ Generalitat de Catalunya, Badalona, 08916 Catalonia, Spain
}

Correspondence should be addressed to Juan Ignacio García; areoso@hotmail.com

Received 15 February 2015; Revised 13 May 2015; Accepted 19 May 2015

Academic Editor: Glenda Gray

Copyright (C) 2015 Juan Ignacio García et al. This is an open access article distributed under the Creative Commons Attribution License, which permits unrestricted use, distribution, and reproduction in any medium, provided the original work is properly cited.

Introduction. The MANGUA cohort is an ongoing multicenter, observational study of people living with HIV/AIDS in Guatemala. The cohort is based on the MANGUA application which is an electronic database to capture essential data from the medical records of HIV patients in care. Methods. The cohort enrolls HIV-positive adults $\geq 16$ years of age. A predefined set of sociodemographic, behavioral, clinical, and laboratory data are registered at entry to the cohort study. Results. As of October 1st, 2012,21697 patients had been included in the MANGUA cohort (median age: 33 years, $40.3 \%$ female). At enrollment $74.1 \%$ had signs of advanced HIV infection and only $56.3 \%$ had baseline CD 4 cell counts. In the first 12 months after starting antiretroviral treatment $26.9 \%(n=3938)$ of the patients were lost to the program. Conclusions. The implementation of a cohort of HIV-positive patients in care in Guatemala is feasible and has provided national HIV indicators to monitor and evaluate the HIV epidemic. The identified percentages of late presenters and high rates of LTFU will help the Ministry to target their current efforts in improving access to diagnosis and care.

\section{Introduction}

Currently, in Guatemala the HIV epidemic is concentrated in most-at-risk populations, with prevalence between 4 and $15 \%$ among commercial sex workers and $11.5-18.3 \%$ among men who have sex with men. HIV prevalence among other vulnerable groups is $18 \%$ in people with tuberculosis (TB),
$13 \%$ in prison populations, and $3.3 \%$ among youth at social risk [1-3]. During the period 2001-2005, with the support from Médecins Sans Frontières, four hospitals were providing antiretroviral treatment (ART) in Guatemala. In 2004, in alignment with the World Health Organization (WHO) 3-5 strategy and with financial aid from the Global Fund to fight AIDS, tuberculosis, and Malaria (the Global Fund), 
TABLE 1: Standardized variables collected in MANGUA.

MANGUA study code, date of enrollment, gender, date of birth, country of origin, province of residence, ethnicity, religion, marital status, educational level, employment status, name of UAI, previous treatment, casualty date, casualty cause, cause of death, informed consent signed, vulnerability group, transmission group, probable year of infection, sexual orientation, and condom use.

Sociodemographic and behavioral variables

Biochemistry and hematology variables, HIV rapid test, HIV ELISA test, serologies for toxoplasma,

Laboratory variables cytomegalovirus, histoplasma, cryptococcus, HBV, HCV, HSV, and syphilis. Sputum-smear microscopy for tuberculosis.

Clinical variables WHO clinical stage, date of visit, type of visit, date of next scheduled visit, CD4 count, CD8 count, HIV RNA viral load, AIDS-related events, AIDS-defining events, and other coinfections.

Treatment variables ART type, prophylaxis type, other treatments, initiation and stop date ${ }^{*}$, reason for regime change ${ }^{*}$, and adverse effects*.

HBV: human hepatitis B virus; HCV: human hepatitis C virus; HSV: human herpes virus simplex types I and II. UAI: Unidad de atención integral; ELISA: enzyme-linked immunosorbent assay; RNA: ribonucleic acid; and ART: antiretroviral treatment.

${ }^{*}$ For each type of treatment.

the Ministerio de Salud Pública y Asistencia Social (MSPAS) started to implement specialized hospital-based HIV unitsUnidades de Atención Integral (UAIs)-across the country; therefore, extending ART coverage and increasing access to care for people living with HIV/AIDS (PLHIV) [4-6]. UAIs are administered by minimal essential staff: a physician, a nurse, a laboratory technician, and a social worker. Currently 16 UAI offer combination antiretroviral therapy (cART) free of charge (Figure 1).

In a collaborative effort between the National AIDS Program (PNS) of the MSPAS and the Fundació Sida $i$ Societat (FSiS), a Catalan non-governmental organization working in global health, the HIV electronic medical record (EMR) application, MANGUA, was developed in 2006 to be implemented in all the UAIs of Guatemala. The purpose of the MANGUA application was to facilitate clinical management and provide data for national HIV indicators to the MSPAS. The MANGUA application is registered in Catalonia, Spain, and was designed and piloted with the support of the HIV department at the Clínica Familiar Luis Ángel García in Guatemala City. The use of the MANGUA application rapidly expanded along with the roll-out and scale-up of UAIs. MANGUA is a database application structured in query language (SQL server 2008 R2, Microsoft, Redmond, WA 98052). It was developed as an EMR to capture demographic, behavioral, clinical, and laboratory information for HIVpositive individuals enrolled in health care services in the UAIs $[1,7]$.

The MANGUA cohort is an ongoing multicenter, prospective observational study based on the data captured in the MANGUA application; its main objective is to generate outputs to improve both public health and clinical practice related to HIV/AIDS in Guatemala.

\section{Methods}

Currently, 14 of the 16 current UAIs participate in the cohort. 21697 HIV-positive adults $\geq 16$ years of age are enrolled. Data are collected by the medical staff of the UAIs following the national ethical guidelines. The cohort includes $74.3 \%$ of the cumulative number of HIV-positive patients reported to the national health authorities in $2012(n=29211)$ [5].
Patients are eligible to enter the MANGUA cohort when there is confirmation of a positive HIV rapid test either with an enzyme-linked immunosorbent assay (ELISA) or a second highly specific rapid test. Discordant results are confirmed by Western blot. Patient informed consent is obtained before performing any HIV test. Participants are recruited at the UAI at HIV diagnosis, referred from primary and secondary level health care centers, or voluntary counseling and testing sites. HIV testing is repeated at the UAI for confirmation. Local policy regarding informed consent for HIV testing is applied.

Data were retrospectively collected from 2001 to 2006, and then, prospectively collected from 2006 onwards. In each UAI there is at least one data entry manager who registers routine health care data obtained from paper medical records into the MANGUA application. Retrospective clinical data dates back to the time of the patient's initial HIV diagnosis or when the clinician first visited the patient. The database is regularly updated to contain follow-up status.

Data are anonymized and sent periodically to Unidad de Apoyo Técnico (UAT) at the FSiS, and to the National Health Care Database (SIGSA), where data are checked for quality before returning to the UAI. All data are stripped of names and other personal identification and a unique MANGUA study code is assigned to each participant to protect confidentiality.

Every two months an interdisciplinary quality control team composed of UAT staff and data managers from PNS visits each UAI to perform source-to-database data audits. At these visits the quality control team selects at random a proportional sample of participants' medical records, such as clinical and laboratory reports, and MANGUA data are reviewed against this original documentation [8].

To guarantee the continual improvement of data validity, UAT data managers generate lists of potential errors (i.e., initiation of ART before HIV diagnosis, etc.) and check for inconsistencies and missing data [1,9]. A MANGUA data dictionary and user manual was developed and distributed among UAI personnel. Descriptive analyses are approved by the PNS and MSPAS. A predefined set of sociodemographic, behavioral, clinical, and laboratory data are recorded at first entry to the cohort study and subsequent visits (Table 1). 


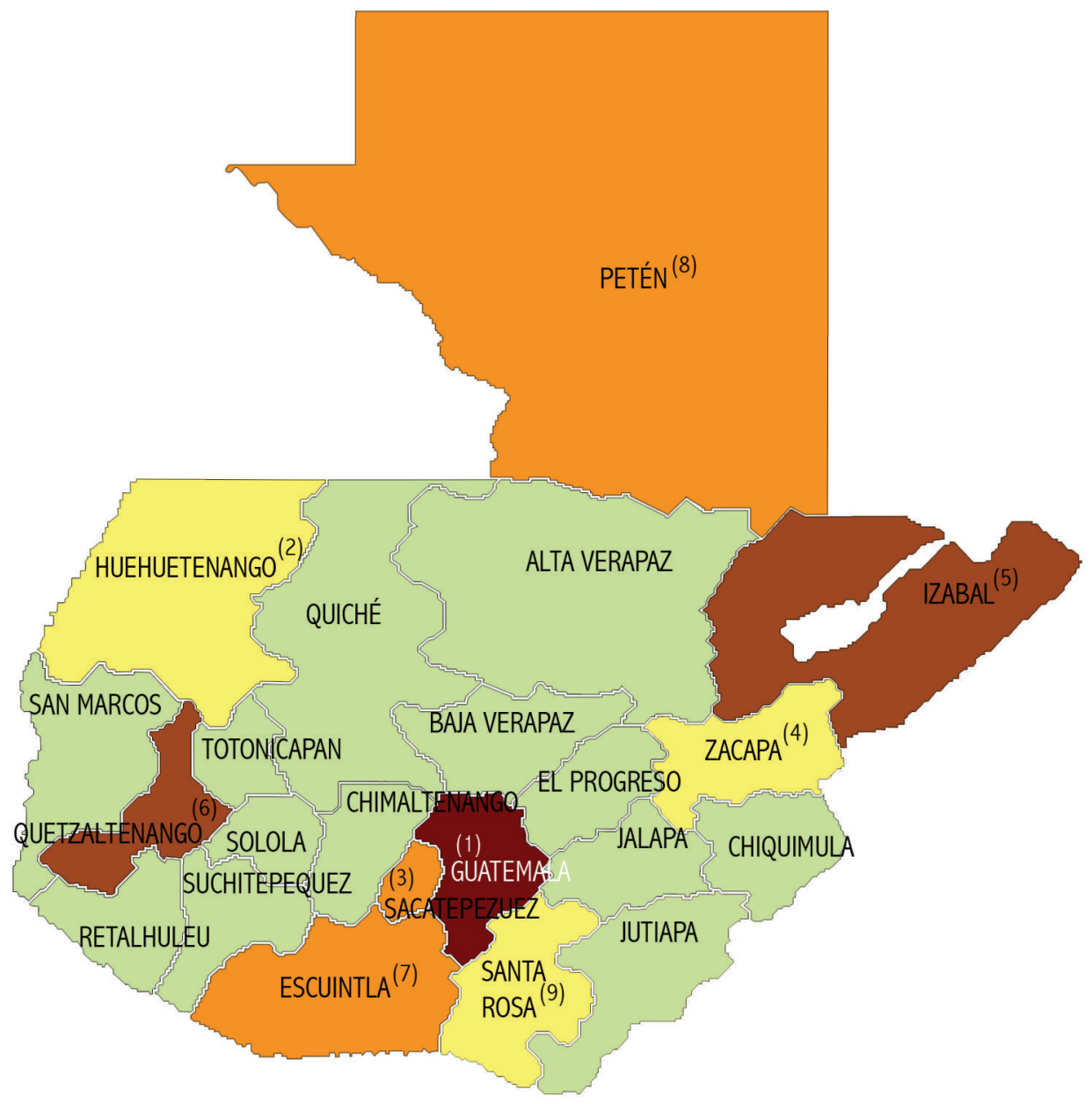

Number of patients by department

\begin{tabular}{l|l}
$5001-12500$ & $0-500$ \\
$1001-5000$ & Departments without UAI \\
$501-1000$ &
\end{tabular}

Number of UAIs and number of patients attended at each UAI by Guatemalan department

(1) Guatemala, 4 UAIs: Clínica Familiar Luis Ángel García, San Juan de Dios Hospital $(n=3887)$. Infectious Diseases Clinic, Roosevelt Hospital $(n=7270)$. Hospital del Instituto Guatemalteco de Seguridad Social (IGSS) $(n=1273)$. Sanidad Militar $(n \approx 60$. UAI not participating in MANGUA cohort. Data not included)

(2) Huehuetenango, 1 UAI: Hospital Departamental de Huehuetenango Dr. Jorge Vides Molina $(n=75)$

(3) Sacatepéquez, 2 UAIs: Hospital Nacional Pedro Bethancourt, Antigua $(n=285)$. Hospicio de San José $(n=363)$

(4) Zacapa, 1 UAI: Hospital Nacional Carlos Arana Osorio $(n=415)$

(5) Izabal, 2 UAIs: Hospital Nacional de la Amistad Japón-Guatemala de Puerto Barrios ( $n=1891)$. Hospital Infantil Elisa Martinez $(n \approx 60$. UAI not participating in MANGUA cohort. Data not included)

(6) Quetzaltenango, 3 UAIs: Juan José Ortega de Coatepeque National Hospital, Coatepeque $(n=3252)$. Hospital Regional de Occidente San Juan de Dios $(n=226)$. Clínica Isaac Cohen Alcache, Hospital Nacional Rodolfo Robles $(n=1174)$

(7) Escuintla, 1 UAI: Hospital Nacional Regional de Escuintla $(n=816)$

(8) Petén, 1 UAI: Hospital Nacional de San Benito Petén $(n=523)$

(9) Santa Rosa, 1 UAI: Hospital Nacional de Cuilapa $(n=247)$

UAI: Unidad de atención integral

FIGURE 1: Number of UAIs and number of patients attended at each UAI by Guatemalan department. 
TABLE 2: Patient characteristics at enrollment in the MANGUA cohort.

\begin{tabular}{|c|c|c|c|c|c|c|c|}
\hline \multirow{2}{*}{ ( $n, \%$ of available data from variable) } & \multicolumn{2}{|c|}{ Total } & \multicolumn{2}{|c|}{ Men } & \multicolumn{2}{|c|}{ Women } & \multirow{2}{*}{$P$ value* } \\
\hline & $n$ & $\%$ & $n$ & $\%$ & $n$ & $\%$ & \\
\hline \multicolumn{8}{|l|}{ Gender $(n=21697,100 \%)$} \\
\hline \multicolumn{8}{|l|}{ Age in years $(n=21629,99.7 \%)$} \\
\hline Median, IQR & $33(27-42)$ & & & & & & \multirow{4}{*}{$<0.0001$} \\
\hline $16-24$ & 3660 & 16.8 & 1616 & 44.5 & 2013 & 55.5 & \\
\hline $25-49$ & 15079 & 69.1 & 9182 & 61.4 & 5779 & 38.6 & \\
\hline$\geq 50$ & 3073 & 14.1 & 2106 & 68.6 & 964 & 18.6 & \\
\hline \multicolumn{8}{|l|}{ Ethnic group ( $n=12172,56.1 \%)$} \\
\hline Ladino & 9842 & 80.9 & 5881 & 59.8 & 3961 & 40.2 & \multirow{2}{*}{0.91} \\
\hline Indigenous & 2330 & 19.1 & 1395 & 59.9 & 935 & 40.1 & \\
\hline \multicolumn{8}{|l|}{ Transmission route $(n=12592,58 \%)$} \\
\hline Heterosexual & 11709 & 92.5 & 6593 & 56.4 & 5102 & 43.6 & \multirow{3}{*}{$<0.0001$} \\
\hline Homosexual & 907 & 7.2 & 844 & 98.1 & 16 & 1.9 & \\
\hline Injection drug users & 40 & 0.3 & 34 & 91.9 & 3 & 8.1 & \\
\hline $\begin{array}{l}\text { Advanced HIV at enrollment }{ }^{* *}(n= \\
10892,50.2 \%)\end{array}$ & 4168 & 38.3 & 2778 & 66.7 & 1390 & 33.3 & $<0.0001$ \\
\hline \multicolumn{8}{|l|}{$\begin{array}{l}\text { CD } 4 \text { cells } / \mathrm{mL} \text { at enrollment }(n=12219 \text {, } \\
56.3 \%)\end{array}$} \\
\hline Median, IQR & $131(46-279)$ & & & & & & \multirow{4}{*}{$<0.0001$} \\
\hline$\geq 350$ & 2129 & 17.4 & 956 & 44.9 & 1173 & 55.1 & \\
\hline $200-349$ & 2311 & 18.9 & 1203 & 52.1 & 1108 & 47.9 & \\
\hline$<200$ & 7779 & 63.7 & 5208 & 66.9 & 2571 & 33.1 & \\
\hline \multicolumn{8}{|l|}{$\begin{array}{l}\text { cART status at enrollment }(n=8919 \text {, } \\
41.1 \%)\end{array}$} \\
\hline Naive & 7981 & 89.5 & 4793 & 60.1 & 3188 & 39.9 & \multirow[t]{2}{*}{0.01} \\
\hline Previous treatment & 938 & 10.5 & 550 & 58.6 & 388 & 41.4 & \\
\hline $\begin{array}{l}\text { Median (IQR) time in days between HIV } \\
\text { diagnosis and cART initiation in days } \\
(n=13195,60.8 \%)\end{array}$ & $75(27-308)$ & $\mathrm{NA}^{* * *}$ & $70(28-247)$ & NA & $86(26-388)$ & NA & 0.97 \\
\hline
\end{tabular}

Classification of AIDS-defining events is based on WHO criteria [10]. At each quarterly or semiannual follow-up visit, laboratory and clinical data are obtained and updated into the MANGUA database.

2.1. Statistical Analysis. Data were analyzed using STATA software version 11, (StataCorp LP, College Station, TX, USA). Sociodemographic and clinical characteristics at enrollment were compared using Pearson $\chi^{2}$ test for categorical variables and Student's $t$-test for continuous variables.

\section{Results}

As of October 1st, 2012, 21697 patients had been included in the MANGUA cohort (median age: 33 years, $40.5 \%$ female). The cohort has 45853 person-years of follow-up, with a median follow-up time of 1.1 years. Percentages of patients with available data on the following variables are as follows: transmission route (58\%), cART status at enrollment (41.1\%), advanced HIV status at enrollment (50.2\%), and CD4 cell counts at enrollment (56.3\%). Among these patients with available data most, 92.9\% (11709/12 592), were infected by heterosexual relations. At enrollment, 89.3\% (8169/9144) were naive and $74.1 \%(12075 / 16300)$ had signs of advanced HIV infection (CD4 cell counts $<350$ cells $/ \mathrm{mm}^{3}$ or WHO clinical stage 3 or 4$)$ [10]. Only 56.3\% (12 219/21697) had baseline CD 4 cell counts, of whom $63.7 \%$ (7782/12 222) had CD4 cell counts $<200$ cells $/ \mathrm{mm}^{3}$ (Table 2 ).

During follow-up, 67.4\% (14616/21697) started ART of which $98 \%$ started cART. At cART initiation, the median CD4 cell count was $126 \mathrm{cell} / \mathrm{mm}^{3}$ (Interquartile range [IQR]: 47240). The treated cohort has 41741 person-years of follow-up and a median follow-up time of 2.3 years. 
Retention analysis was based on the definition described elsewhere [11, 12]. Retention proportions of living HIVpositive adults on active cART were calculated at 12 and 24 months after cART initiation. Pre- and postdecentralization periods should be differentiated. During the 2001-2005 predecentralization period the average retention was $84 \%$ and $79.7 \%$ at 12 and 24 months, respectively, with 2095 patients starting cART. For the 2006-2011 postdecentralization period, the average retention was $65.3 \%$ and $55.5 \%$ at 12 and 24 months, respectively, with 11265 patients starting cART.

Overall, for the 11-year period 2001-2011, the average retention proportion was $73.8 \%$ and $61.5 \%$ at 12 and 24 months, respectively.

Attrition analysis was adapted from definitions described elsewhere $[11,13]$ and was defined as episodes of discontinuation of cART at the end of a follow-up period for the following reasons: death, loss to follow-up (LTFU), or stopping ART for any other reason while remaining in care. LTFU included the following two situations [14]. The first situation applies when patients are permanently LTFU for a 12-month period during which any additional patient visits disqualified them as permanently LTFU. In this situation, a differentiation was made between patients with no follow-up, defined as those that did not return to the clinic after the first visit scheduled for cART initiation, and patients with LTFU after starting cART, defined as those who start cART and are lost to followup for at least 180 days after the last visit scheduled. The second situation applies to patients LTFU who later reentered follow-up, defined as those who start cART and return for at least one visit after which the patient is not seen in the clinic for $\geq 180$ days after the last visit scheduled but reappears at the clinic from at least day 181 onwards.

In order to calculate the global LTFU rate, the analysis took into account that a single patient might have had more than one episode of LTFU. Thus, during 41741 person-years of follow up, 8572 episodes of LTFU (including cART stops for other reasons), were recorded with a rate of 20.5 per 100 person-years, very close to the rates seen elsewhere [14].

Attrition rates at 6- and 12-month follow-up period were studied; the 6-month interval was chosen to be consistent with the LTFU definition used; the 12-month interval was used to analyze the evolution of LTFU episodes and to be able to compare LTFU outcomes with retention rates. In the first 6 months after starting cART, $19 \%(n=2785)$ of patients were lost to the program: 575 patients $(20.6 \%)$ did not return to the clinic after cART initiation (no follow-up), 1568 (56.3\%) were LTFU (including cART stops for other reasons), and 642 (23.1\%) died. In the first 12 months after starting cART $26.9 \%$ $(n=3938)$ of patients were lost to the program: 618 patients (15.7\%) did not return to the clinic after cART initiation (no follow-up), 2510 (63.7\%) were LTFU (including cART stops for other reasons), and 810 (20.6\%) died.

Future measurements will include an analysis of LTFU risk factors, late diagnosis, and incidence of opportunistic infections by CD 4 cell counts. It is also necessary to measure death outcomes in patients starting cART and the incidence of side effects of cART.

\section{Discussion}

This is the largest cohort of HIV-positive people compiled in Central America and the cohort has a wide representation of the total HIV population registered throughout Guatemala. The MANGUA cohort has a nationwide, population-based design, with long-term follow-up. National scale-up of cART has resulted in a considerable number of patients enrolled in HIV treatment and care for HIV prevention and control in a country constrained by limited resources.

The MANGUA application was implemented soon after the scale-up of UAIs; in 2004 only 4 UAIs existed in the country; in 2008, 12 UAIs were already functioning. Scale-up of UAIs was possible because of international financial aid, mainly from the Global Fund, and the commitment of the MSPAS to decentralize HIV care in Guatemala. This scenario facilitated the acceptability of an EMR by UAI professionals, because it allowed systematization and harmonization of data collection and analysis. Comprehensive training activities were targeted to professionals, physicians, nurses, and data entry personnel, on how to use the MANGUA application. The implementation of the MANGUA cohort has been successful and has proven to be useful for both clinicians and the PNS through the provision of data for key HIV indicators to monitor the HIV epidemic in Guatemala. The MANGUA application has been integrated within the SIGSA web information system, which is crucial to ensure sustainability and strengthen HIV monitoring and surveillance in Guatemala.

Nevertheless, only approximately half of the patients registered in the cohort have data available on CD4 cell counts at baseline, partly due to under-notification and the fact that only some UAIs have available CD4 cell count analyzers. Among those with baseline data on CD4 cell counts, as much as $82.6 \%$ do not present for HIV testing until advanced symptoms of HIV infection (CD4 cell counts $<350 / \mathrm{mm}^{3}$ ). Late presenters are often more ill, have a higher mortality risk, and are less likely to respond to treatment after initiation [15-18].

According to the Guatemalan National Guidelines on HIV Diagnosis and Treatment, adults with WHO clinical stage disease 3 or 4 or CD 4 cell counts $<350$ cells $/ \mathrm{mm}^{3}$ are eligible for cART [19]. However, $65.1 \%$ of those eligible patients do not start cART treatment and many of them are LTFU (Figure 2).

Delayed cART initiation has been reported to be the major cause of death and associated morbidity in HIV/AIDS patients $[20,21]$. This fact, together with late presentation for HIV diagnosis and care and high LTFU, remains a significant challenge to efficiently prevent morbidity and mortality outcomes. It is worrisome that $30.1 \%$ (2582/8572) of patients considered LTFU return to the hospital after having interrupted ART for at least 6 months, which increases their risk of developing drug resistance or death [22-24]. Efforts should be focused on identifying reasons for LTFU with strategies to minimize it, such as creating interdisciplinary teams to investigate its underlying causes, proposing solutions that have shown to be effective in other contexts $[25,26]$, and expanding treatment access points in order to reduce time spent travelling to the clinic. 

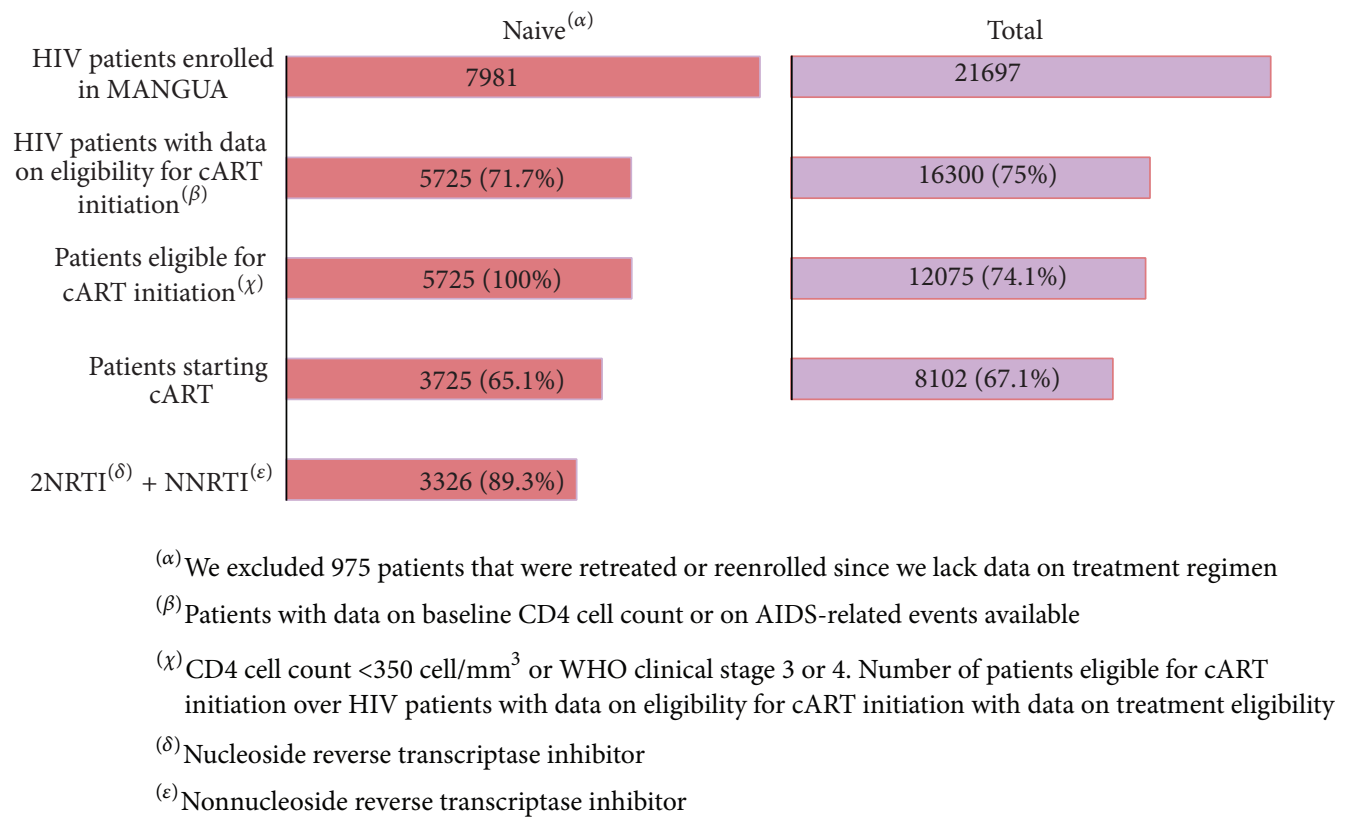

FIGURE 2: Spectrum of cART initiation in patients enrolled in MANGUA cohort.

Retention analysis reveals that the postdecentralization period has less retention proportions than the predecentralization period. This might be explained by different factors; first, the number of HIV-positive patients starting cART in the postdecentralization period is 5.4 times higher than in the predecentralization period; and second, recently created UAIs might have less capacity to provide integrated care to HIV-positive patients, lacking the experience to effectively implement adherence and follow-up interventions.

\section{Conclusions}

Today, the MANGUA HIV cohort is a powerful tool for monitoring and evaluation as well as for research on HIVpositive patients in respect to (i) epidemiologic patterns of the HIV epidemic, (ii) delayed access to cART and burden of opportunistic infections, (iii) risk factors for LTFU and late diagnosis, and (iv) long term cART outcomes.

The strengths of this cohort include its size and the representativeness of data collected from almost all registered HIV patients in Guatemala gathered from 14 of the 16 UAIs. As a main strength, data within MANGUA covers the spectrum of engagement in care, including late HIV diagnosis, suboptimal linkage to and retention in HIV care, insufficient use of antiretroviral therapy, and suboptimal adherence to therapy [27].

In Guatemala, the AIDS surveillance system is composed of several sources of information that are not yet linked. As a result, the system is highly heterogenic and complex, making it difficult to extract data for decision making [28]. Information on quality of the AIDS surveillance system is lacking, that is, whether it is representative of the whole country and updated in a timely manner and its level of completeness and accuracy. The MANGUA database is contributing to the harmonization of HIV national surveillance data. In 2012, it gained recognition from national stakeholders and UNAIDS Guatemala as the official HIV application system. Some limitations should be noted. The MANGUA cohort has not yet reached $100 \%$ of registered HIV-positive patients declared to the MSPAS (29 211). Moreover, neither the MSPAS nor MANGUA has the ability to register HIVpositive patients from private health care centers throughout Guatemala; thus, selection bias in the MANGUA cohort might be a limitation.

The cohort is dependent on the quality of data entered by data entry managers from written medical records; therefore, long standing and skilled staff are required to maintain the quality of the clinical data, which is not always feasible. We attempt to validate clinical data and provide feedback to the UAIs in order to minimize errors and improve data recording. Nonetheless, data omissions, duplicated patient records, and incomplete and out-of-date laboratory and sociodemographic information might occur. In fact, there is a high proportion of missing data in key variables such as CD4 cell counts at baseline, treatment status at enrollment, transmission route of infection, and clinical stage category. This limits the ability to draw conclusions from the entire cohort because of data representativeness and selection bias due to differences in characteristics of patients with data available compared to patients without complete data available. In most UAIs it is not possible to maintain computerized records; clinical data are collected for administrative purposes rather than for clinical ones. Although the MSPAS is currently working through this, there is no standardized paper-based instrument shared in all UAIs to collect the essential variables recorded in MANGUA cohort, which would help in homogenizing data collection. 
The MANGUA cohort is not linked with the national registry of deaths; consequently, there is little information on AIDS-related deaths, and mortality rates may be underestimated. In addition, TB and HIV services are not well integrated in Guatemala. TB screening of HIV-positive patients is poorly performed in UAIs and patients are referred to the primary health care center for TB preventive chemotherapy and treatment. Thus, data entry managers from UAIs lack information on TB treatment outcomes on HIV patients, hampering data entry into the MANGUA application from the UAIs, and underestimating $\mathrm{TB}$ associated incidence among HIV-positive people.

Nevertheless, the two main limitations are: first, the MANGUA application is not used directly by medical staff as an EMR in most UAIs, rather data are collected initially on paper and then digitalized. The process of informatization of the UAIs in the country may, on a medium-term basis, contribute to correct this situation. Second, high rates of LTFU may introduce selection bias due to differences in follow-up. UAI decentralization needs to be consolidated to partly overcome this problem, the stabilization of the staff in peripheral UAIs being one of its main challenges, which will assure the continuum of care and facilitate adherence to treatment.

The MANGUA cohort aims to put Guatemalan HIV/ AIDS research on a par with other international collaborations in the field. This will be achieved through the development of a nationally and internationally recognized policy-relevant program of research in HIV therapy and public health and through the establishment of training and research opportunities to graduate students, postdoctoral fellowships, and clinicians across the country.

Our efforts to improve research dissemination to physicians and PLHIV as well as to improve knowledge and translational research will contribute to global recognition of the MANGUA cohort. Over the past 6 years, we have expanded our capacity to recruit participants, collect, extract, and analyze data, and engage clinicians, policy stakeholders, and funders. We are committed to share and promote MANGUA research findings to help shape programs and policy that will improve the lives of PLHIV in Guatemala and elsewhere.

\section{Disclosure}

The members of the MANGUA Cohort Study Group include the following: Executive Committee: Eduardo Arathoon, Carlos Mejía, Zonia Pinzón, Enrique Ponce, Elsy Camey, and Jordi Casabona; Scientific Committee: Elsy Camey, Juan Ignacio García, Meritxell Sabidó, Luis A. Prieto, Mikhail Nikiforov, Luis Ardani, and Jordi Casabona, from Fundació Sida i Societat, Escuintla, Guatemala; Eduardo Arathoon, Blanca Samayoa, and Erwin Argueta, from Clínica Familiar Luis Ángel García, San Juan de Dios Hospital, Asociación de Salud Integral, Guatemala City, Guatemala; Carlos Rodolfo Mejía, Rodolfo Pinzón, Johana Samayoa, Mirci Romero, and Ericka Boror, from Infectious Diseases Clinic, Roosevelt Hospital, Guatemala City, Guatemala; Ernesto Ponce and Pedro Rizzo, from the National Programme for Prevention and Control of STI and HIV/AIDS (PNS), Guatemala City, Guatemala; José Fernando Ortiz and Edwin Leslie Cambranes Morales, from Hospital del Instituto Guatemalteco de Seguridad Social (IGSS), Guatemala City, Guatemala; Vilma Reyes, from Hospital Departamental de Huehuetenango Dr. Jorge Vides Molina, Huehuetenango, Guatemala; Carolina Zacarías from Hospital Nacional Pedro Bethancourt, Antigua, Sacatepéquez, Guatemala; Maudi González from Hospicio de San José, Sacatepéquez, Guatemala; Claudia Mazariegos from Hospital Nacional Carlos Arana Osorio, Zacapa, Guatemala; Carlos Eduardo Chajón and Marco Antonio Tobar from Hospital Nacional de la Amistad Japón-Guatemala de Puerto Barrios, Izabal, Guatemala; Luis Roberto Santa Marina de León, Gladys Sajche, and Ingrid Azucena from Juan José Ortega de Coatepeque National Hospital, Coatepeque, Quetzaltenango, Guatemala; Diana Mazariegos from Hospital Regional de Occidente San Juan de Dios, Quetzaltenango, Guatemala; César Augusto López-Téllez and Janet Ikeda from Clínica Isaac Cohen Alcache; Hospital Nacional Rodolfo Robles, Quetzaltenango, Guatemala; Aura Méndez from Hospital Nacional Regional de Escuintla, Guatemala; Mario Baldizón from Hospital Nacional de San Benito Petén; Petén, Guatemala; Alba Contreras from Hospital Nacional de Cuilapa, Santa Rosa, Guatemala.

\section{Conflict of Interests}

All authors declare that they have no conflict of interests.

\section{Authors' Contribution}

All authors have made substantial contributions to design, analysis, and interpretation of data and have been involved in drafting and revising the paper critically for important intellectual content and have given final approval of the version to be published.

\section{Acknowledgments}

The authors would like to thank all participants of the Guatemalan HIV cohort study, particularly the staff at the UAIs for their continuous support, enthusiasm, and commitment to improve lives of PLHIV. They thank Virginia Isern, Lidia Serra, Luis Arriaga, and Jordi Figuerola for their early contribution to the design of the MANGUA application. They thank Jordi Morell for preparing paper figures. They thank Adriana Smith for improving the use of English in the paper. This work was supported by the Catalan Agency for Development (ACCD), the Spanish Agency for International Development Cooperation (AECID), the City Hall of Barcelona, The Global Fund to Fight AIDS, tuberculosis and Malaria, The Unites States Agency for International Development, through PASCA (USAID/PASCA), and FSiS. 


\section{References}

[1] M. Forster, C. Bailey, M. W. G. Brinkhof et al., "Electronic medical record systems, data quality and loss to follow-up: survey of antiretroviral therapy programmes in resource-limited settings," Bulletin of the World Health Organization, vol. 86, no. 12, pp. 939-947, 2008.

[2] USAID, Guatemala HIV/AIDS Health Care Profile, USAID, Santa Catarina Pinula, Guatemala, 2010.

[3] WHO, Guatemala Summary Country Profile for HIV/AIDS Treatment Scale-Up, World Health Organization, Geneva, Switzerland, 2005.

[4] USAID, Decentralizing Antiretroviral Treatment Services at Primary Health Care Facilities, USAID, 2010.

[5] MSPAS/CNE/PASCA, Guatemalan HIV Statistics Guatemala, 2012.

[6] MSPAS and PNS, National Strategic Plan 2006-2010. Prevention and Control of STIs and HIV/AIDS, MSPAS, PNS, 2006.

[7] H. S. F. Fraser, C. Allen, C. Bailey, G. Douglas, S. Shin, and J. Blaya, "Information systems for patient follow-up and chronic management of HIV and tuberculosis: a life-saving technology in resource-poor areas," Journal of Medical Internet Research, vol. 9, no. 4, p. e29, 2007.

[8] S. N. Duda, B. E. Shepherd, C. S. Gadd, D. R. Masys, and C. C. McGowan, "Measuring the quality of observational study data in an international HIV research network," PLoS ONE, vol. 7, no. 4, Article ID e33908, 2012.

[9] C. W. Whitney, B. K. Lind, and P. W. Wahl, "Quality assurance and quality control in longitudinal studies," Epidemiologic Reviews, vol. 20, no. 1, pp. 71-80, 1998.

[10] WHO, WHO Case Definitions of HIV for Surveillance and Revised Clinical Staging and Immunological Cassification of HIV-related Disease in Adults and Children, WHO, 2007.

[11] S. Rosen, M. P. Fox, and C. J. Gill, "Patient retention in antiretroviral therapy programs in sub-Saharan Africa: a systematic review," PLoS Medicine, vol. 4, no. 10, article e298, 2007.

[12] UNAIDS, Global AIDS Response Progress Reporting Guidelines. Construction of Core Indicators for Monitoring the 2011 Political Declaration on HIV/AIDS, UNAIDS, 2012.

[13] M. W. G. Brinkhof, F. Dabis, L. Myer et al., "Early loss of HIVinfected patients on potent antiretroviral therapy programmes in lower-income countries," Bulletin of the World Health Organization, vol. 86, no. 7, pp. 559-567, 2008.

[14] J. Zhou, J. Tanuma, R. Chaiwarith et al., "Loss to followup in HIV-infected patients from Asia-pacific region: results from TAHOD," AIDS Research and Treatment, vol. 2012, Article ID 375217, 10 pages, 2012.

[15] M. May, J. Gill, C. Lewden et al., "Causes of death in HIV1-infected patients treated with antiretroviral therapy, 19962006: collaborative analysis of 13 HIV cohort studies," Clinical Infectious Diseases, vol. 50, no. 10, pp. 1387-1396, 2010.

[16] Opportunistic Infections Project Team of the Collaboration of Observational HIV Epidemiological Research in Europe (COHERE) in EuroCoord, "CD4 cell count and the risk of AIDS or death in HIV-Infected adults on combination antiretroviral therapy with a suppressed viral load: a longitudinal cohort study from COHERE," PLoS Medicine, vol. 9, no. 3, Article ID e1001194, 2012.

[17] R. S. Hogg, B. Yip, K. J. Chan et al., "Rates of disease progression by baseline CD4 cell count and viral load after initiating tripledrug therapy," Journal of the American Medical Association, vol. 286, no. 20, pp. 2568-2577, 2001.
[18] S. P. Koenig, L. A. Rodriguez, C. Bartholomew et al., "Long-term antiretroviral treatment outcomes in seven countries in the Caribbean," Journal of Acquired Immune Deficiency Syndromes, vol. 59, no. 4, pp. e60-e71, 2012.

[19] MSPAS and PNS, Guatemalan National Guidelines for Antiretoviral Therapy and Opportunistic Infections Treatment, MSPAS, Guatemala, North America, 2011.

[20] F. Zhang, Z. Dou, Y. Ma et al., "Effect of earlier initiation of antiretroviral treatment and increased treatment coverage on HIV-related mortality in China: a national observational cohort study," The Lancet Infectious Diseases, vol. 11, no. 7, pp. 516-524, 2011.

[21] J. A. Sterne, M. May, D. Costagliola et al., "Timing of initiation of antiretroviral therapy in AIDS-free HIV-1-infected patients: a collaborative analysis of 18 HIV cohort studies," The Lancet, vol. 373, no. 9672, pp. 1352-1363, 2009.

[22] P. T. Nieuwkerk, M. A. G. Sprangers, D. M. Burger et al., "Limited patient adherence to highly active antiretroviral therapy for HIV-1 infection in an observational cohort study," Archives of Internal Medicine, vol. 161, no. 16, pp. 1962-1968, 2001.

[23] P. R. Harrigan, R. S. Hogg, W. W. Y. Dong et al., "Predictors of HIV drug-resistance mutations in a large antiretroviralnaive cohort initiating triple antiretroviral therapy," Journal of Infectious Diseases, vol. 191, no. 3, pp. 339-347, 2005.

[24] M. Charurat, M. Oyegunle, R. Benjamin et al., "Patient retention and adherence to antiretrovirals in a large antiretroviral therapy program in Nigeria: a longitudinal analysis for risk factors," PLoS ONE, vol. 5, no. 5, Article ID e10584, 2010.

[25] H. Tweya, D. Gareta, F. Chagwera et al., "Early active followup of patients on antiretroviral therapy (ART) who are lost to follow-up: the 'Back-to-Care' project in Lilongwe, Malawi," Tropical Medicine and International Health, vol. 15, supplement 1, pp. 82-89, 2010.

[26] S. T. Alamo, G. J. Wagner, P. Sunday et al., "Electronic medical records and same day patient tracing improves clinic efficiency and adherence to appointments in a community based HIV/AIDS care program, in Uganda," AIDS and Behavior, vol. 16, no. 2, pp. 368-374, 2012.

[27] E. M. Gardner, M. P. McLees, J. F. Steiner, C. Del Rio, and W. J. Burman, "The spectrum of engagement in HIV care and its relevance to test-and-treat strategies for prevention of HIV infection," Clinical Infectious Diseases, vol. 52, no. 6, pp. 793$800,2011$.

[28] J. Barczyk, J. García, and J. Casabona, "The potential contribution of small-scale intervention projects in the field to the national health information system for HIV and sexually transmitted infections: a case study of a multilevel intervention in Guatemala," International Journal of Public Health, vol. 55, no. 6, pp. 693-699, 2010. 


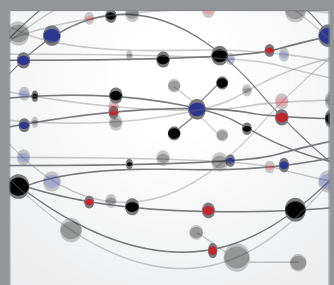

The Scientific World Journal
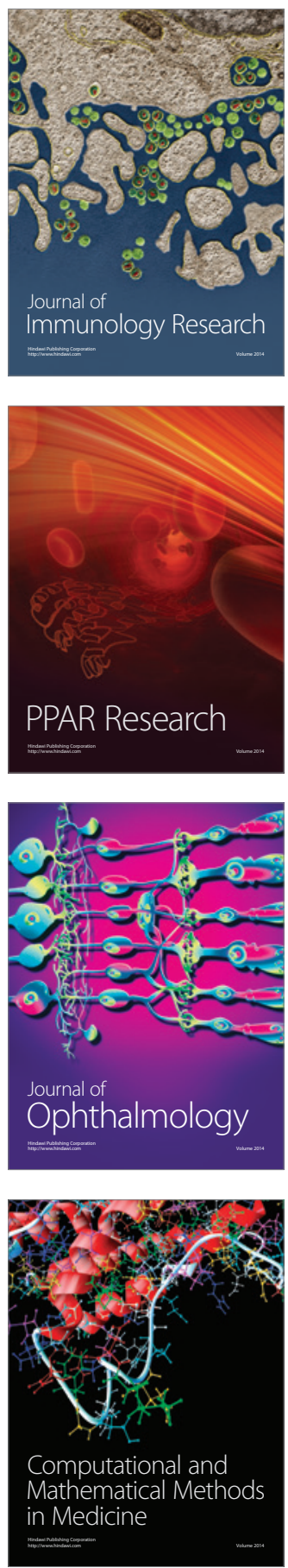

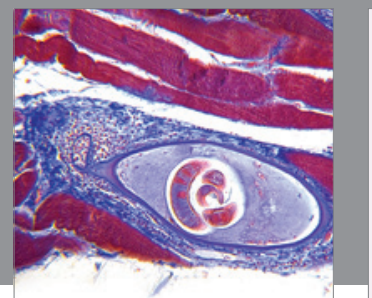

Gastroenterology

Research and Practice
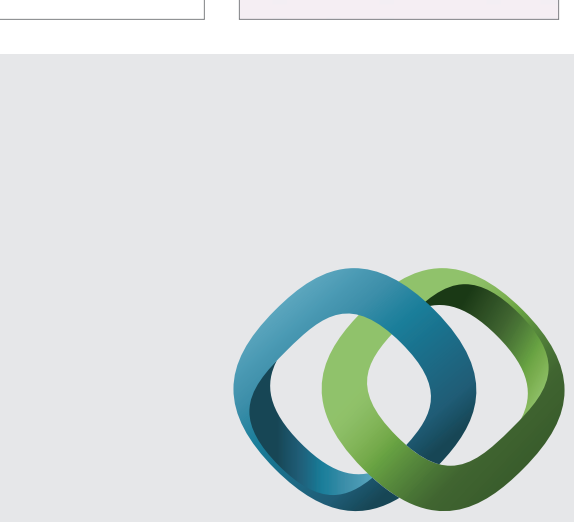

\section{Hindawi}

Submit your manuscripts at

http://www.hindawi.com
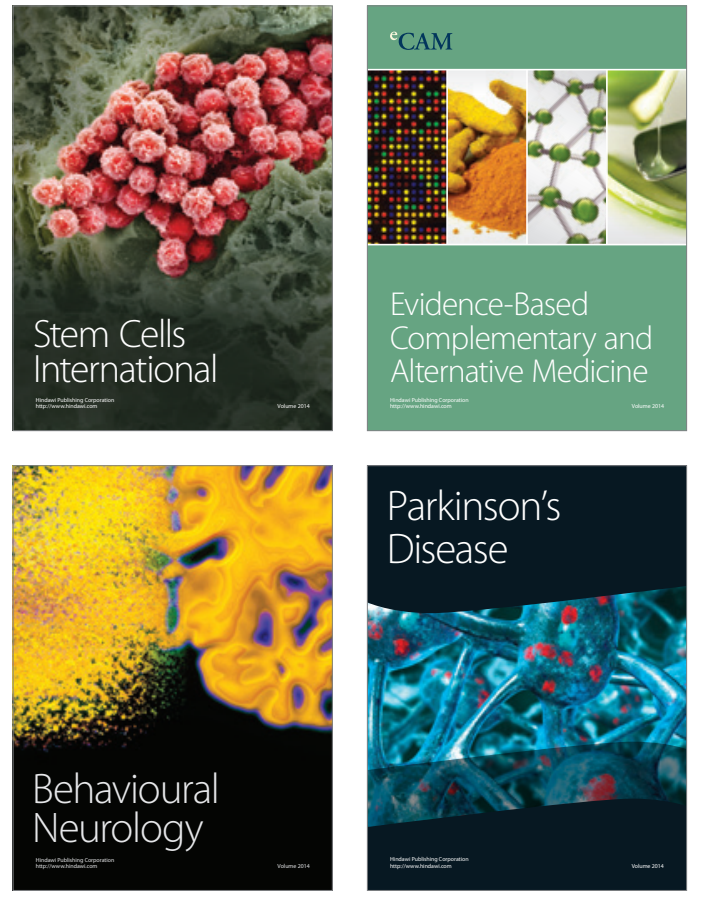
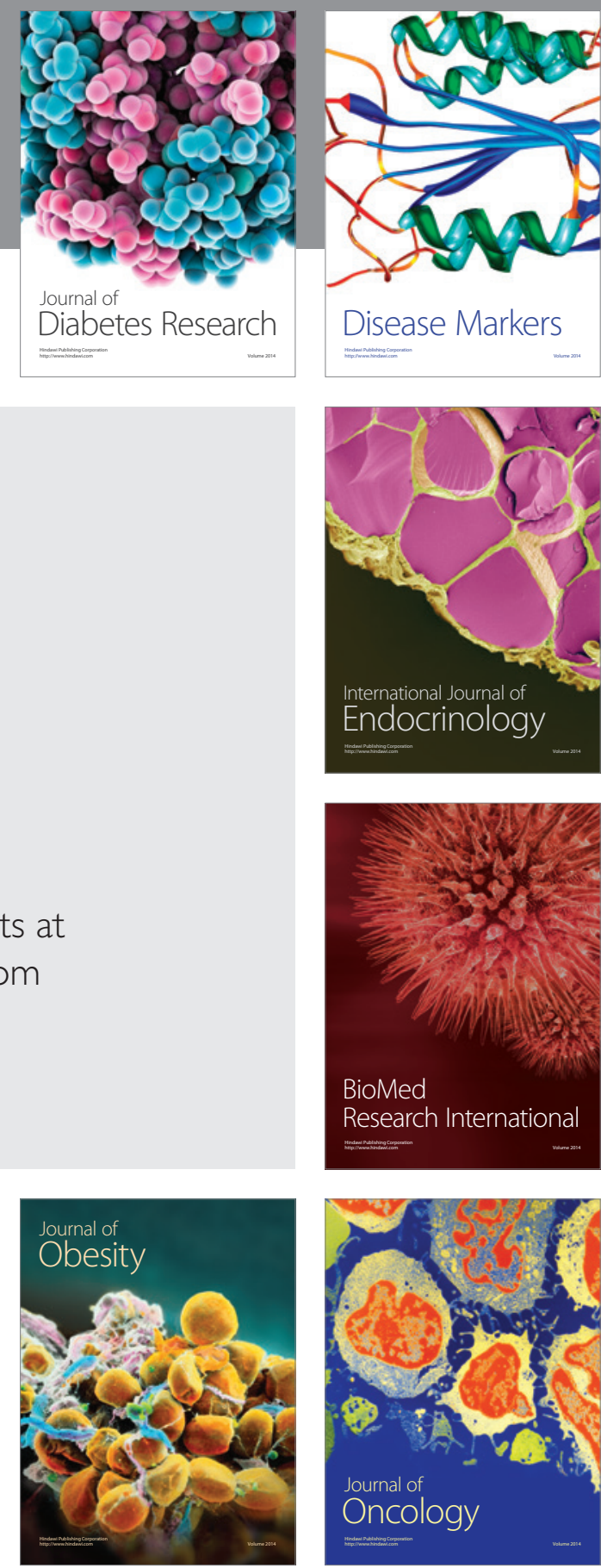

Disease Markers
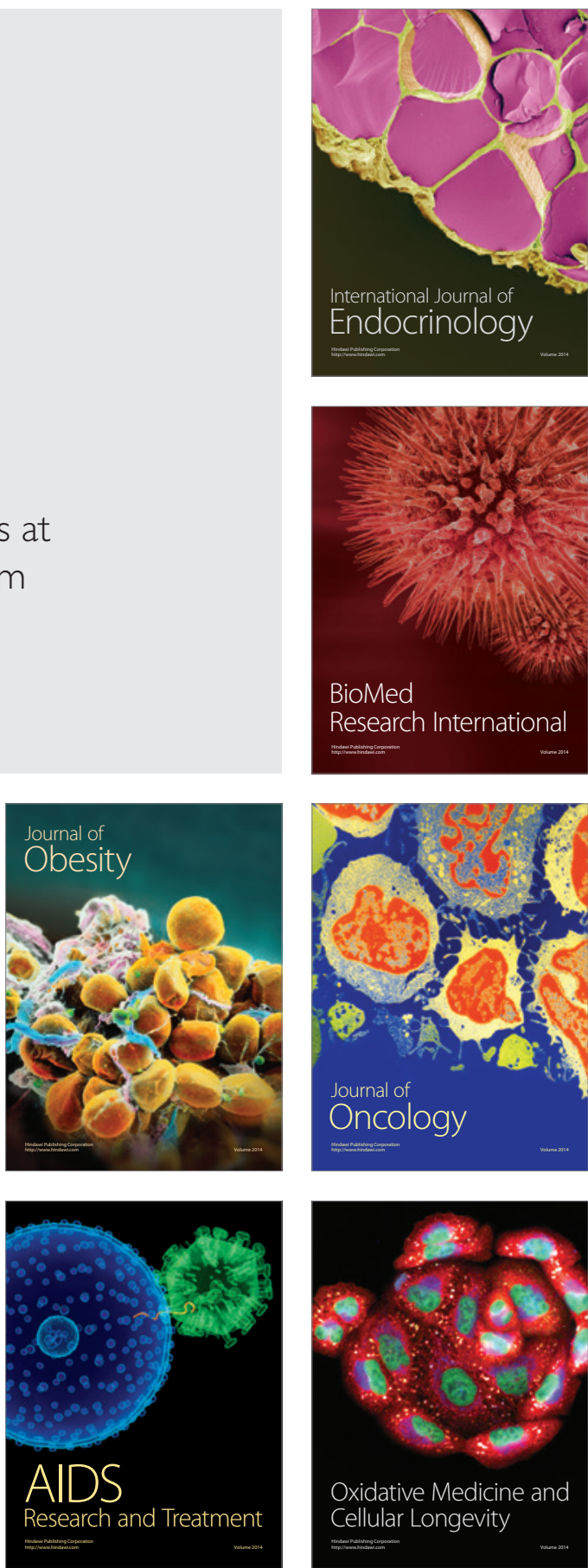